\title{
Outcome of repeated thrombolysis in Debrecen Thrombolysis Database
}

Klára Fekete MD $\mathrm{PhD}^{1}$, Sándor Márton $\mathrm{PhD}^{2}$, László Csiba MD PhD DHAS ${ }^{1}$, István Fekete $\mathrm{MD} \mathrm{PhD}^{1}$

${ }^{1}$ Department of Neurology, Faculty of Medicine, University of Debrecen, Hungary

${ }^{2}$ Department of Sociology and Social Politics, Institute of Political Sciences and Sociology, Faculty of Arts and Humanities, University of Debrecen, Hungary

Address correspondence to:

Dr. István Fekete

Department of Neurology

University of Debrecen

Debrecen

Móricz Zs. krt. 22.

H-4032, Hungary

Tel: +36 52255255 , Fax: +36 52255255

E-mail: fekete@med.unideb.hu

Highlights: Outcome of repeated thrombolysis

Word count: 3237

Key words: recurrent ischemic stroke, thrombolysis database, intravenous thrombolysis, repeated thrombolysis, longterm outcome, symptomatic intracranial hemorrhage 
Email addresses for authors:

Klára Fekete: feketek@med.unideb.hu

Sándor Márton: marton.sandor@arts.unideb.hu

László Csiba: csiba@med.unideb.hu

István Fekete: fekete@med.unideb.hu 


\begin{abstract}
Introduction

Recombinant tissue plasminogen activator (rtPA) is an efficient therapy of acute ischemic stroke. The risk of a recurrent ischemic stroke is high. This prospective single center study was to assess whether the repeated rtPA-treatment is beneficial for acute stroke patients or not.
\end{abstract}

\title{
Methods
}

All thrombolysed patients' data at the Department of Neurology, University of Debrecen have been recorded in the Debrecen Thrombolysis Database (DTD) since 2004. We identified 21 patients with repeated thrombolysis. Stroke severity by the NIH stroke scale score (NIHSSS) and imaging findings by the ASPECT score were evaluated on admission and one day later. The modified Rankin Scale (mRS) at 3 months and case fatality at one year were evaluated. Comparing first and second thrombolyses, we screened for bleeding and allergic reactions to determine safety.

\section{Results}

Within the 27-month median time, 18 patients were thrombolysed twice, with complete follow-up. In recurrent stroke patients diabetes mellitus, congestive heart failure and anticoagulation were more common. Admission cholesterol levels were also decreased. After the first and second treatments, 24-hour-NIHSSS were $3(1 ; 6)$ and $7(1 ; 10)$, respectively. At three months, good outcome was significantly higher after the first treatment than those of DTD, with no differences between two attempts. There was little difference in three-month and one-year outcomes, regardless of laterality - ipsilateral or contralateral hemisphere - in recurrent strokes. One patient had non-symptomatic intracranial bleeding after repeated rtPAtreatment.

\section{Discussion}

Recurrent rtPA treatment may be safe and effective in patients who have mild or moderate residual symptoms after the index stroke. 


\section{Introduction}

Recombinant tissue plasminogen activator (rtPA) is an efficient therapy of acute ischemic stroke $[1,2,3,4,5]$. Nevertheless it has been an established fact that the risk of a recurrent ischemic stroke is high, especially within five years. Death and severe disability are more probable in case of a recurrent stroke. However, different publications have reported development of antibodies against the recombinant protein in animal models [6;7] and humans alike $[8,9,10]$. These facts raise the question of allergy to rtPA and the neutralizing antibodies against the rtPA, at the second attempt, possibly influencing the treatment unfavourably [10].

The aim of this study was to assess whether repeated rtPA treatment is beneficial for recurrent acute ischemic stroke patients or not (because of possible desensitization). We were interested whether there are more side effects (e.g. allergic reaction, haemorrhage) by the second attempt than by the primary treatment.

\section{Methods}

\section{Subjects}

Started on $1^{\text {st }}$ January 2004, the Thrombolysis Database includes all thrombolysed patients' data from the Department of Neurology, University of Debrecen with a catchment area of approximately 600,000 inhabitants and 600-700 acute stroke hospitalizations per year. The time window from symptom onset was three hours for intravenous (IV) treatment until October 2008, and 4.5 hours afterwards. The Regional Research Ethics Committee of the University of Debrecen approved intra-arterial (IA) use of rtPA with a 6-hour time-window. Altogether we identified 21 patients who had undergone repeated thrombolysis. Three of those did not complete follow up but the 18 patients who did, were enrolled in the study. They were divided into three groups as follows: first thrombolysed (I.), second thrombolysed (II.), and both groups were compared with a pooled group of all patients in the Debrecen Thrombolysis Database (DTD all) of 369 patients. The DTD was chosen for comparison since it was a well-defined homogenous group [11].

\section{Database}

We created a database recording details such as age, gender, time of stroke onset, time of arrival in the hospital, time of the CT scan result, time of administering rtPA, previous medical history (hypertension, diabetes mellitus, atrial fibrillation, heart failure, self reported smoking and alcohol consumption habits), on-admission stroke severity (including the National Institute of Health Stroke Scale Score - NIHSSS) [12], prestroke modified Rankin 
Scale score (mRS) [13, 14], systolic and diastolic blood pressure before the initiation of rtPA administration, serum glucose level, INR, APTT, cholesterol, triglyceride, on-admission CT/CTA scan, and, if performed, digital subtraction angiography, and a follow-up (24 hours \pm 2 hours) CT scan. We registered the NIHSS Score at 24 hours, medications for secondary prevention, mRS at three months, and survival status at one year. The study was approved by the Regional Research Ethics Committee of the University of Debrecen.

\section{Imaging}

Non-contrast computed tomography (CT) was performed on admission. Arterial occlusion (trunk or at least one branch of any large artery) was identified by CTA. CT was repeated one day after treatment and in case of clinical deterioration. The Alberta Stroke Programme Early CT Score (ASPECTS) was determined unblinded to patient characteristics and was stratified to $\leq 7$ (Group I-severe) and above 7 (Group II-mild) [15, 16]. Hemorrhagic infarction (HI) or parenchymal hematoma $(\mathrm{PH})$ was defined according to the European Cooperative Acute Stroke Study $[17,18]$. We used three definitions for symptomatic intracerebral hemorrhage (SICH): the SITS, ECASS and NINDS criteria [1, 17, 19].

\section{Treatment}

IV treatment was administered according to the EUSI and ESO guidelines of 2003 and 2008, respectively [20, 21]. Among the twice thrombolysed patients one underwent IA treatment, and he received a total of 30mg rtPA until opening of the artery. IA use of rtPA was approved by the Regional Research Ethics Committee of the University of Debrecen.

\section{Outcomes}

The NIHSSS and case fatality were evaluated the day after admission. Outcome assessment was performed using mRS at three months and outcomes were dichotomized into favourable (mRS 0-2) and unfavourable (mRS>2 points) [22]. Survival status was evaluated at one year.

\section{Statistical analysis}

Statistical analysis was carried out using the SPSS for Windows 19.0 (SPSS Inc. Chicago, USA). The categorical variables were assessed with Pearson $\chi 2$ test.

Significant differences were considered if $\mathrm{p}<0.05$.

\section{Results}

\section{Prestroke characteristics}

The median time between two thrombolytic therapies was 27 months (5-67). Among the patients receiving treatment $55.5 \%$ were males. The prevalence of hypertension was similar in all of the groups. Prestroke diabetes mellitus was more common in case of recurrent 
therapy (Group II) than at the first attempt (Group I) $(p=0.087)$ or in the DTD group $(p<0.05)$. Prestroke congestive heart failure was slightly but not significantly increased in Group II ( $\mathrm{p}=$ 0.423). Prestroke anticoagulation was significantly higher $(\mathrm{p}<0.05)$ in group II than in Group I or DTD ( $\mathrm{p}<0.05)$. Of course, all of the patients in Group II had a history of stroke (Table 1). On admission, cholesterol levels were much lower in Group II, compared with Group I and Group DTD $(\mathrm{p}<0.05)$. Triglyceride levels were in the normal range in all groups.

\section{Treatment characteristics}

There was no significant difference in the onset to treatment time between the two attempts (140 minutes group I and 136 minutes Group II) (Figure 1).

Based on systolic and diastolic blood pressure, there was no significant difference among the groups. Blood sugar levels before treatment were also similar in all groups.

The severity of stroke on admission was similar in both attempts on admission: first treatment $9(7 ; 13)$, second treatment: $9(6 ; 14)$ (Table 1). The median 24-hour-NIHSSS was $3(1 ; 6)$ after the first treatment, and $7(1 ; 10)$ after the recurrent rtPA treatment. After the first thrombolysis the 24-hour improvement was better than after the repeated treatment ( 2 points).

\section{Outcome}

Progression could be detected in recurrent stroke compared to the status after the first treatment. The improvement after the recurrent stroke was smaller in NIHSSS at 24 hours, than after the index stroke. The median 24-hour-NIHSSS was $3(1 ; 6)$ after the first treatment and $7(1 ; 10)$ after the recurrent rtPA treatment.

The outcome at 24 hours was significantly better after the first treatment than after second thrombolysis $(\mathrm{p}<0.05)$ or in all DTD group $(\mathrm{p}<0.05)$ Even the second treatment had a significant benefit compared with the DTD group $(\mathrm{p}<0.05)$ (Table 1).

We characterized new ischemic lesions using ASPECT Score. At 24 hours, the average ASPECT score was 10 for Group I and 9 for Group II, i.e. 9 and 8 points, respectively. Comparing the groups in the study it can be stated, that ASPECTS at 24 hours at the first treatment was better, but it was still very good even if it was compared with DTD all (Figure 2).

At three months, favourable outcomes scored higher, i.e. poor outcomes and deaths were significantly lower in both Group I, Group II than in DTD all ( $p<0.05$ I.-DTD; $p=0.068$ II.DTD) However, there was no difference between Groups I and II ( $\mathrm{p}=0.338$ ) (Figure 3). After the first rtPA, all patients were alive at one year as expected but after the second one, three patients were dead at one year $(27.7 \%)$. At one year there was a significant difference 
between the outcome of first thrombolysis and the DTD group $(\mathrm{p}<0.05)$; but the other comparisons were not significant (II.-DTD p=0.311; I.-II. $\mathrm{p}=0.07$ ).

Outcome at three months and at one year did not differ significantly if the recurrent stroke affected the ipsilateral or contralateral hemisphere (Figure 4).

\section{Safety data}

Among the patients with complete follow up, no haemorrhagic transformation, SICH (symptomatic intracerebral hemorrhage) was detected either after the index rtPA treatment or after recurrent thrombolysis. However, one patient whose follow up was not completed had a haemorrhagic transformation (not SICH). Minor bleeding (subconjunctival, gingival and tongue bleeding), occurred after the second thrombolysis in four cases but there was no need to interrupt their treatment. No allergic reaction or angioneurotic edema occurred.

At three months, mortality rates in Group II and DTD all were $11.1 \%$ and $22.8 \%$, while at one year the relevant data were found at $27.7 \%$ and $35.5 \%$, respectively (Figure 3).

\section{Discussion}

Unfavourable outcome (disability and death) increases in case of recurrent stroke [23]. Despite its beneficial effect, recurrent thrombolysis has been reported in only few publications $[23,24]$.

The patients in the study had several risk factors such as diabetes mellitus, atrial fibrillation, congestive heart failure, and, of course, stroke when rethrombolysed. Nevertheless we assume, that many of these risk factors did not appear as new, but had been there silently and undiscovered prior to the first stroke. This study was not designed to prove this assumption, since further research is required to verify this theory. However, it must be highlighted that significantly lower cholesterol levels show the importance of secondary stroke prevention (statin therapy). The fact that systolic blood pressure exceeded $140 \mathrm{mmHg}$ even in the group with recurrent stroke shows the shortcomings of care.

Although all patients were aware of the symptoms of stroke at the second time, they did not arrive at our department earlier. The median door-to-needle time was within the 60 minutes' golden hour as advised in the latest guidelines [25].

Most patients after the index stroke had mild symptoms, but at the onset of recurrent stroke, patients had moderate symptoms. In recurrent stroke, the ASPECTS status at 24 hours was worse compared to that in the first treatment. 
Contrary to our expectations the outcome was similar if the stroke appeared either in the ipsilateral or in the contralateral hemisphere. Of course, this can also be attributed to small sample size.

Death ratio was similar to those thrombolysed only once even after the recurrent rtPA treatment. The outcome at 24 hours and at three months was worse after the second thrombolysis, but the number of previous diseases was higher. Only one patient had nonsymptomatic intracranial bleeding after the second rtPA treatment. Low rate of intracranial bleeding could be due to careful patient selection. With recruiting patients with more severe neurological symptoms and ASPECTS the bleeding ratio would probably be higher. Repeated thrombolysis among our patients was also safe.

Our study has several limitations. The number of the patients with repeated thrombolysis is small, and our study is possibly underpowered to draw conclusions regarding effectiveness and safety. By examining our data of twice thrombolysed patients, we found that they had mild or moderate symptoms after the first thrombolysis. Despite the above, this study might have its value. Similarly to our data all publications in this field include a small sample, showing the rarity of recurrent treatment [23]. The advantage of this study may be that all 21 patients were treated and controlled after stroke at the same department.

\section{Conclusion}

Although drawing conclusions has its limitations we think that recurrent rtPA treatment may be safe and effective in patients who have mild or moderate residual symptoms after the index stroke. Definitely, a study of a larger sample size is necessary to allow for further conclusions.

\section{Conflict of interest:}

K.F. as assistant national coordinator for SITS EAST received travel funding for conferences (2008-2010) (Modest)

S.M.: none

L.CS.: is the national coordinator for SITS EAST, and has received fees from Boehringer Ingelheim for giving lectures (Modest)

I.F.: has received fees from Boehringer Ingelheim for giving lectures (Modest)

Funding: none

\section{Acknowledgements:}

The authors thank the medical staff taking part in the treatment. 


\section{References}

1. The National Institute of Neurological Disorders and Stroke rt-PA Stroke Study Group. Tissue plasminogen activator for acute ischemic stroke. N Engl J Med 1995;333:1581-1587.

2. Kwiatkowski TG, Libman RB, Frankel M, et al. Effects of tissue plasminogen activator for acute ischaemic stroke at one year. National Institute of Neurological Disorders and Stroke Recombinant Tissue Plasminogen Activator Stroke Study Group. N Engl J Med 1999;340:1781-1787.

3. Hacke W, Kaste M, Bluhmki E, et al. Thrombolysis with Alteplase 3 to 4.5 hours after acute ischemic stroke. N Engl J Med 2008;359:1317-1329.

4. Wardlaw JM, Murray V, Berge E, et al. Recombinant tissue plasminogen activator for acute ischaemic stroke: an updated systematic review and meta-analysis. Lancet 2012;379:2364-2372.

5. Ahmed N, Kellert L, Lees KR, et al. SITS Investigators. Results of intravenous thrombolysis within 4.5 to 6 hours and updated results within 3 to 4.5 hours of onset of acute ischemic stroke recorded in the Safe Implementation of Treatment in Stroke International Stroke Thrombolysis Register (SITS-ISTR): an observational study. JAMA Neurol 2013;70:837-844.

6. Katsutani N, Yoshitake S, Takeuchi H, et al. Immunogenic properties of structurally modified human tissue plasminogen activators in chimpanzees and mice. Fundam Appl Toxicol 1992;19:555-562.

7. Zwickl CM, Hughes BL, Piroozi KS, et.al. Immunogenicity of tissue plasminogen activators in rhesus monkeys: antibody formation and effects on blood level and enzymatic activity. Fundam Appl Toxicol 1996;30:243-254. 
8. Reed BR, Chen AB, Tanswell P, et al. Low incidence of antibodies to recombinant human tissue-type plasminogen activator in treated patients. Thromb Haemost 1990;64:276-280.

9. Cugno M, Cicardi M, Colucci M, et al. Non neutralizing antibodies to tissue type plasminogen activator in the serum of acute myocardial infarction patients treated with the recombinant protein. Thromb Haemost 1996;76:234 -238.

10. Sauer R, Huttner HB, Breuer L, et al. Repeated Thrombolysis for Chronologically Separated Ischemic Strokes A Case Series. Stroke 2010;41:1829-1832.

11. Fekete K, Márton S, Tóth J, et al. Predictors of long-term outcome after intravenous and intraarterial rtPA treatment in the Eastern Hungarian Thrombolysis Database. $\mathrm{J}$ Stroke Cerebrovasc Dis 2015:117-24.

12. Lyden P, Brott T, Tilley B, et al. Improved reliability of the NIH Stroke Scale using video training. NINDS TPA Stroke Study Group. Stroke 1994;25:2220-2226.

13. van Swieten JC, Koudstaal PJ, Visser MC, et al. Interobserver agreement for the assessment of handicap in stroke patients. Stroke 1988;19:604-607.

14. Quinn TJ, Dawson J, Walters MR., et al. Reliability of the Modified Rankin Scale: A Systematic Review. Stroke 2009;40:3393-3395.

15. Barber PA, Demchuk AM, Zhang J, et al. Validity and reliability of a quantitative computed tomography score in predicting outcome of hyperacute stroke before thrombolytic therapy. ASPECTS Study Group. Alberta Stroke Programme Early CT Score. Lancet 2000;355:1670-1674.

16. Pexman JH, Barber PA, Hill MD, et al. Use of the Alberta Stroke Program Early CT Score (ASPECTS) for assessing CT scans in patients with acute stroke. AJNR Am J Neuroradiol 2001;22:1534-1542. 
17. Larrue V, von Kummer R, Müller A, et al. Risk factors for severe hemorrhagic transformation in ischemic stroke patients treated with recombinant tissue plasminogen activator: a secondary analysis of the European-Australasian Acute Stroke Study (ECASS II). Stroke 2001;32:438-441.

18. Berger C, Fiorelli M, Steiner T, et al. Hemorrhagic transformation of ischemic brain tissue: asymptomatic or symptomatic? Stroke 2001;32:1330-1335.

19. Wahlgren N, Ahmed N, Dávalos A, et al. SITS-MOST investigators. Thrombolysis with alteplase for acute ischaemic stroke in the Safe Implementation of Thrombolysis in Stroke-Monitoring Study (SITS-MOST): an observational study. Lancet 2007;369:275-282.

20. The European Stroke Initiative Executive Committee and the EUSI Writing Committee. European Stroke Initiative Recommendations for Stroke Management Update 2003. Cerebrovasc Dis 2003;16:311-337.

21. The European Stroke Organisation (ESO) Executive Committee and the ESO Writing Committee. Guidelines for management of ischaemic stroke and transient ischaemic attack. Cerebrovasc Dis 2008;25:457-507.

22. Thomassen L, Waje-Andreassen U, Naess H. Early ischemic CT changes before thrombolysis: The influence of age and diabetes mellitus. Ther Clin Risk Manag 2008;4:699-703.

23. Cappellari M, Moretto G, Bovi P. Repeated intravenous thrombolysis after recurrent stroke. A case series and review of the literature. J Neurol Sci. 2014;345(1-2):181-3.

24. Kushawa S, Malik S, Sarraf G, et al. Thrombolysis in recurrent stroke- Beyond guidelines: A case report. J Stroke Cerebrovasc Dis 2014:23(8);e407-e408.

25. Jauch EC, Saver JL, Adams HP Jr, et al. AHA/ASA Guideline. Guidelines for the early management of patients with acute ischemic stroke. A guideline for healthcare 
professionals from the American Heart Association/American Stroke Association. Stroke 2013;44:870-947. 


\section{Figure legends}

Table 1 Prestroke parameters and basic parameters of the rtPA treatment

Figure 1 Logistic data. Onset to treatment time by I. rtPA treatment (median) 140 min, II. rtPA treatment (median) $136 \mathrm{~min}$

Figure 2 ASPECTS at 24 hours. /p =0.027 I.-DTD; $\mathrm{p}=0.012$ II.-DTD;I.-II./

Figure 3 Outcome at 3 months (A) /p =0.009 I.-DTD; $p=0.068$ II.-DTD; I.-II. $p=0.338 /$ and at one year (B) /p =0.008 I.-DTD; $\mathrm{p}=0.311$ II.-DTD; I.-II. $\mathrm{p}=0.07 /$

Figure 4 Outcome at 3 months (modified Rankin Scale Score) and at one year (case fatality) after recurrent thrombolysis depending on whether the recurrent stroke affected the ipsilateral or contralateral hemispheres 